\title{
microRNA expression profiles in human colorectal cancers with brain metastases
}

\author{
ZHENYANG LI* , XIAODONG GU*, YANTIAN FANG, JIANBIN XIANG and ZONGYOU CHEN \\ Department of General Surgery, Huashan Hospital, Fudan University, Shanghai 200040, P.R. China
}

Received August 19, 2011; Accepted November 21, 2011

DOI: $10.3892 / \mathrm{ol} .2011 .497$

\begin{abstract}
The present study aimed to identify microRNA (miRNA) expression profiles associated with brain metastases of colorectal cancers. We conducted miRNA expression profiling of 3 primary colorectal cancers and 3 brain-metastatic carcinomas using Agilent Human miRNA Microarrays. Quantitative real-time reverse transcription-polymerase chain reaction (RT-PCR) was employed to validate the results obtained from the miRNA microarray analysis. Overexpression of miR-145, miR-1, miR-146a, miR-576-5p, miR-126*, HS287, miR-28-5p, miR-143, miR-199b-5p, miR-199a-5p, miR-10b, miR-22, miR-133b, miR-145*, miR-199a, miR-133a, miR-125b and downregulation of miR-31 and HS170 were observed in brain-metastatic carcinomas. Quantitative RT-PCR experiments with miR-125b confirmed the expression patterns we found in our microarray experiments. miRNAs are differentially expressed between colorectal cancers and matched brain-metastatic carcinomas. The miRNA variation trend is quite different in the process of metastasis compared to that of carcinogenesis. These miRNAs may therefore serve as potential diagnostic markers and therapeutic targets for colorectal cancers with brain metastases.
\end{abstract}

\section{Introduction}

Colorectal cancer (CRC) is one of the most common digestive tract malignancies worldwide. The incidence of CRC is lower in China than in Western countries, but has increased in recent years due to changes in lifestyle and nutritional habits. CRC is currently one of the most serious threats to human health in China (1). Over the past decade, newer and more effective systemic therapies have been applied in advanced

Correspondence to: Dr Zongyou Chen or Dr Jianbin Xiang, Department of General Surgery, Huashan Hospital, Fudan University, 12 Wulumuqi Middle Road, Shanghai 200040, P.R. China

E-mail: zongyouc@sohu.com; xjbzhw@163.com

*Contributed equally

Key words: brain metastasis, colorectal cancer, microarray, microRNA
CRC patients. Active chemotherapeutic agents (specifically capecitabine, oxaliplatin and irinotecan) and biological drugs (such as cetuximab and bevacizumab) have provided further significant benefits (2). Due to marked advances in life prolongation, the liver and lungs are no longer exclusive targets among potential metastatic sites, and the incidence of metastases at previously uncommon sites, such as the brain, is on the increase (3). Brain metastases are relatively rare in CRC, with a reported incidence ranging from $<1$ to $4 \%$. Furthermore, newer neuroimaging modalities have superior accuracy in identifying small ( $<5 \mathrm{~mm}$ ) asymptomatic brain lesions (4). Therefore, CRC with brain metastasis requires careful consideration.

Gene expression profiling in CRC has been extensively studied in recent years. In the majority of studies, differences in gene expression were observed between normal mucosa and CRC tissues. Certain investigators studied metastasis-related genes by comparing CRC and corresponding metastases from the same patients, and found that expression levels of the majority of differentially expressed genes varied moderately $(5,6)$. These findings were in accordance with the theory, proposed by Bernards and Weinberg (7), that there are no genes or genetic changes that are specifically and exclusively involved in the process of metastasis. Instead, the combination of oncogenic alterations, rather than particular events, determines the metastatic genotype of tumor cells early in tumorigenesis.

Mounting evidence indicates that microRNAs (miRNAs) may be key players in the regulation of tumor cell invasion and metastasis (8). miRNAs are a large family of small non-coding RNAs that negatively control gene expression at the mRNA and protein levels. Mature miRNAs comprise 19-25 nucleotides that silence genes through consummate or defective binding to the $3^{\prime}$ untranslated region of the transcript. Microarray studies have revealed that individual miRNAs are capable of affecting the expression of multiple genes, indicating that miRNAs may have pleiotropic effects on cellular processes including cell differentiation, proliferation and apoptosis $(9,10)$. An increasing number of studies have indicated that there are differential miRNA expression profiles between those in cancer tissues and adjacent non-tumorous tissue (11-14). However, few studies of metastasis-related miRNAs have compared CRC and matched metastatic carcinomas.

As has been established, metastasis, the colonization of tumor cells in another site discontinuous from the primary $\mathrm{CRC}$, is the major cause of death for CRC patients (15). To understand the molecular mechanism of CRC with brain 
Table I. Clinicopathological features of 4 paired primary and metastatic CRCs.

\begin{tabular}{|c|c|c|c|c|c|c|c|c|}
\hline \multirow[t]{2}{*}{$\begin{array}{l}\text { Case } \\
\text { No. }\end{array}$} & \multirow[t]{2}{*}{ Gender } & \multirow[t]{2}{*}{ Age } & \multicolumn{2}{|c|}{$\begin{array}{l}\text { Tumor } \\
\text { size, cm }\end{array}$} & \multicolumn{2}{|c|}{$\begin{array}{l}\text { Histological } \\
\text { differentiation }\end{array}$} & \multirow[t]{2}{*}{$\begin{array}{l}\text { Primary } \\
\text { site }\end{array}$} & \multirow[t]{2}{*}{$\begin{array}{l}\text { Metastatic } \\
\text { site }\end{array}$} \\
\hline & & & $\mathrm{P}$ & M & $\mathrm{P}$ & M & & \\
\hline 1 & M & 46 & 4.5 & 2.0 & Poor & Poor & Sigmoid & Right parietal lobe \\
\hline 2 & M & 59 & 6.0 & 3.0 & Moderate & Moderate & Descending & Cerebella \\
\hline 3 & $\mathrm{~F}$ & 56 & 4.0 & 2.5 & Moderate & Poor & Sigmoid & Left frontal lobe \\
\hline 4 & $\mathrm{~F}$ & 54 & 6.0 & 4.0 & Poor & Poor & Ascending & Left frontal lobe \\
\hline
\end{tabular}

$\mathrm{P}$, primary tumor; $\mathrm{M}$, metastatic tumor.

metastases, we used microarrays to directly characterize the molecular basis of metastases. We then selected 3 primary $\mathrm{CRC}$ tissues and matched metastatic carcinomas from the brain, and compared the miRNA expression profiles.

\section{Materials and methods}

Patients and tissue specimens. Primary CRC samples were collected from 4 patients who underwent radical resection of CRC in Huashan Hospital of Fudan University from May 2005 to November 2008. Their matched metastatic carcinomas from the brain were resected within 3 months prior or subsequent to the digestive tract surgery. Craniotomy and radical surgery for CRC were performed in our hospital to allow collection of the samples of CRC and brain-metastatic carcinomas. Tissues were snap-frozen in liquid nitrogen following surgical resection and stored at $-80^{\circ} \mathrm{C}$ until RNA extraction. Clinical information was collected from patient records. None of the CRC patients had received chemotherapy prior to surgery. Written informed consent was obtained from all CRC patients or their guardians. This study was performed according to the Committee for the Conduct of Human Research of Huashan Hospital of Fudan University.

RNA isolation. Total RNA was isolated using the mirVana ${ }^{\mathrm{TM}}$ miRNA Isolation Kit (Ambion, Austin, TX, USA) following the manufacturer's instructions. The RNA quantity was then determined using a Nanodrop 1000 Spectrophotometer (Thermo Scientific, Wilmington, DE, USA). The RNA quality was assessed using the Agilent 2100 Bioanalyzer (Agilent, Santa Clara, CA, USA). The samples were assayed and qualified in triplicate using the miRNA microarray.

miRNA microarray profiling. The input for the Agilent miRNA labeling system was 100 ng total RNA. After dephosphorylation and denaturation, the total RNA was labeled with cyanine 3-pCp and then hybridized to Agilent Human miRNA Microarray V2.0 using the miRNA Complete Labeling and Hyb Kit (Agilent). Following hybridization for 20 h, the slides were washed using the Gene Expression Wash Buffer Kit (Agilent) and scanned using an Agilent Scanner. The images were processed and analyzed with Agilent Feature Extraction Software. The raw data were normalized using quantile normalization and then analyzed in GeneSpring GX software
(zcomSilicon Genetics, Redwood City, CA, USA). Statistical analysis using ANOVA was conducted to compare the differentially expressed miRNAs.

Quantitative real-time reverse-transcription polymerase chain reaction (RT-PCR). A cDNA synthesis was carried out using a miScript Reverse Transcription Kit (Qiagen, Hilden, Germany) according to the manufacturer's instructions. A quantitative PCR was performed using a miScript SYBRGreen PCR Kit (Qiagen). Expression analysis was performed in triplicate for each sample. The small nuclear RNA U6 was used as the normalization control. The miRNA expression level was quantified using the ABI 7300 Sequence Detection System (Applied Biosystems, Foster City, CA, USA).

Statistical analysis. Results are presented as the mean \pm standard deviation. Statistical analyses were carried out using Stata 8.0 software (Stata, College Station, TX, USA). The comparison of miRNA expression between primary CRC and metastatic carcinomas was carried out using Student's t-test. $\mathrm{P}<0.05$ was considered statistically significant. The normalization of data and selection of the differentially expressed miRNAs were performed using GeneSpring GX software.

\section{Results}

Clinicopathological characteristics of paired primary and metastatic CRC. Our 4 primary and matched metastatic CRC pairs were all synchronous. Patient characteristics and other clinical findings are shown in Table I. The time from primary tumor to development of brain metastases was $\leq 3$ months. In Case 1 and Case 4 patients, the colorectal primary was identified after the diagnosis of the brain secondary.

miRNA expression profiling in primary CRC versus matched brain metastases. To investigate the role of miRNAs in brain metastasis of human CRC, we conducted a comprehensive miRNA expression profiling of 3 primary CRC and 3 brain-metastatic carcinomas (cases 1,2,3) using the Agilent Human miRNA Microarray V2.0. This microarray contains 15744 probes representing 723 human miRNAs. A total of 19 miRNAs were differentially expressed between primary CRC and brain-metastatic carcinomas. In the brain-metastatic carcinomas, 2 miRNAs were downregulated and 17 miRNAs 
Table II. MiRNAs correlated to brain metastasis of human CRC.

\begin{tabular}{lcc}
\hline miRNA & $\begin{array}{c}\text { Regulation in brain- } \\
\text { metastatic carcinomas }\end{array}$ & $\begin{array}{c}\text { Chromosome } \\
\text { location }\end{array}$ \\
\hline miR-199a & Up & $1,19,9$ \\
miR-133a & Up & 20,18 \\
miR-145 & Up & 5 \\
miR-143 & Up & 5 \\
miR-10b & Up & 2 \\
miR-1 & Up & 20,18 \\
miR-199a-5p & Up & 1,19 \\
miR-145* & Up & 5 \\
miR-31 & Down & 9 \\
miR-125b & Up & 21,11 \\
miR-133b & Up & 6 \\
miR-22 & Up & 17 \\
HS_170 & Down & 0 \\
miR-126* & Up & 9 \\
miR-146a & Up & 5 \\
miR-28-5p & Up & 3 \\
miR-576-5p & Up & 4 \\
miR-199b-5p & Up & 9 \\
HS_287 & Up & 0 \\
\hline
\end{tabular}

were upregulated (Table II). These results indicate that these 19 miRNAs may play significant roles in the brain metastasis of human CRC. Additionally, hierarchical cluster analysis based on the expression patterns of these 19 miRNAs accurately separated the primary carcinomas from the metastatic ones (Fig. 1).

Validation of microarray data by real-time RT-PCR. To validate the miRNA microarray results, miR-125b was chosen for analysis by real-time RT-PCR in all 4 CRC cases. The real-time RT-PCR results were in concordance with the miRNA microarray analysis results (Fig. 2). Tissues with higher expression levels of miR-125b were from brain-metastatic lesions.

\section{Discussion}

$\mathrm{CRC}$ is the most common cancer in China. The common sites of metastasis in CRC include the lung, liver and draining lymph nodes. Brain metastases are relatively rare in CRC. However, the incidence of metastases to the brain is on the increase, due to effective therapies for $\mathrm{CRC}$, which have provided longer survival times, and more effective neuroimaging techniques. The genesis and treatment of brain metastases from CRC has therefore attracted attention $(16,17)$. We also reviewed CRC patient records between 2001 and 2008 in Huashan Hospital. A total of 28 patients who developed brain metastases were identified (18). Our data indicated that the median survival following surgery of brain secondaries was 9.4 months, which is similar to previous reports of patients with brain metastases from CRC in which the median survival was 8.3 months (19).

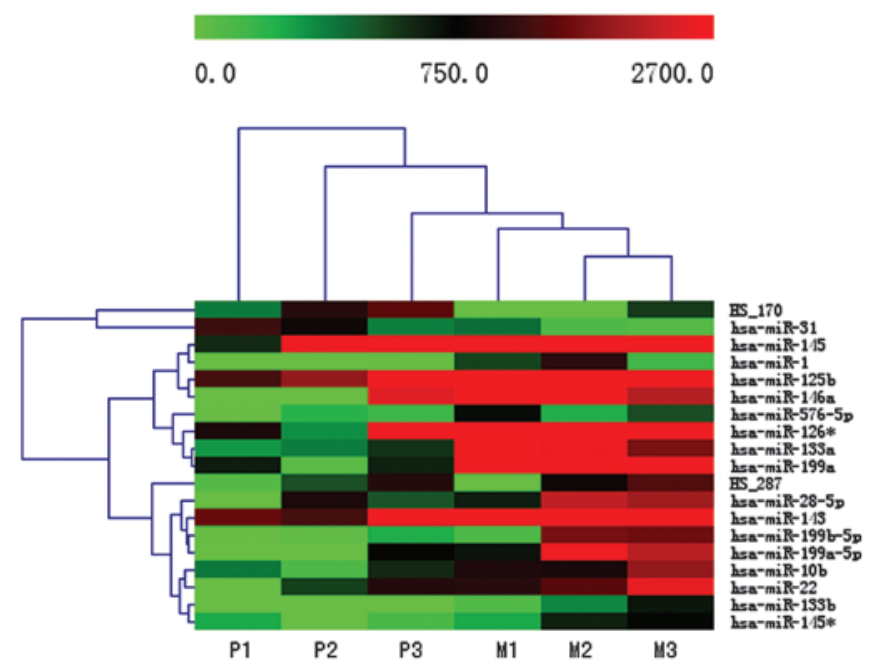

Figure 1. The miRNA expression signature in primary CRC (P) versus the matched brain-metastatic carcinomas (M). The heatmap was based on 19 differentially expressed miRNAs, the expression of which correlated with brain metastasis of human CRC.

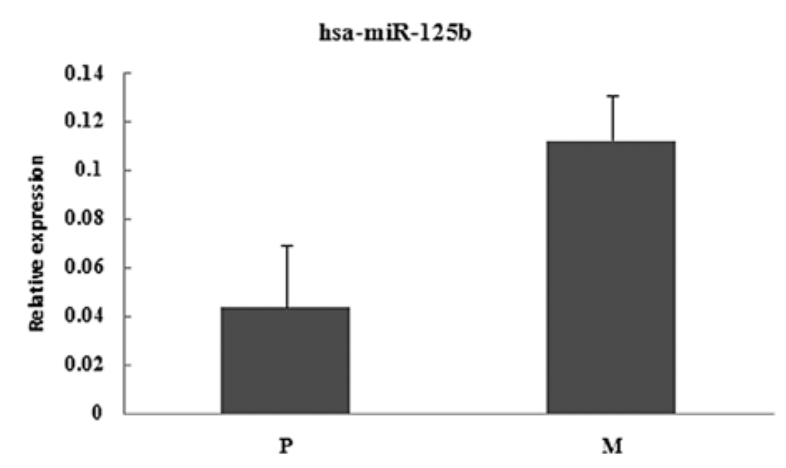

Figure 2. Validation of the differentially expressed miRNAs by real-time RT-PCR. MiR-125b was markedly upregulated in brain metastatic carcinomas (M), but not in primary CRC $(\mathrm{P})$.

It is recommended that a brain scan is performed when lung and/or liver metastases are found, particularly in patients with a rectal or sigmoid colon cancer (4). This finding may lead to earlier diagnosis, amenability to surgical treatment and improved quality of life.

In recent years, advances in molecular biology have markedly expanded our knowledge of the molecular mechanism of metastasis in CRC. Pantaleo et al (20) found that the molecular background of liver metastases was different: EGFR and COX-2 were overexpressed in metachronous and synchronous metastases, respectively. These authors proposed that therapies based on EGFR pathway inhibition may be considered for metachronous metastases (such as cetuximab) and therapies based on the inhibition of angiogenesis crosstalking pathways (such as bevacizumab) may be considered for synchronous metastases. However, gene expression profiling of matched CRC and metastases may reveal a small number of key molecular events. Koehler et al (5) indicated that a specific expression signature was not identified in matching metastases. Koh et al (6) also demonstrated a similar gene expression profile between paired primary and metastatic CRC. These 
results are consistent with Bernards and Weinberg's theory that there are no genes or genetic changes that are specifically and exclusively involved in the process of metastasis (7).

It has been established that the potential of a tumor cell to metastasize depends on numerous factors. Mounting evidence has indicated that miRNAs may be key players in the regulation of tumor cell invasion and metastasis (8). miRNAs are small non-coding RNAs that serve as negative regulators of gene expression. Through interactions with the 3' untranslated region of mRNA by partial sequence homology, miRNAs cause gene silencing by mRNA degradation or translation repression. Given the unique feature of their targeting, each single miRNA may have over a hundred targets, and, thus, a large number of protein-coding genes may be regulated by miRNAs (21). Huang et al (22) performed a comparison of the miRNA profile of tumor-adjacent non-cancerous colonic samples in CRC with lymph node metastasis with those without lymph node metastasis. Their results indicated that hsa-miR-129* and hsa-miR-137 were significantly differentially expressed. Wang et al (11) found that 14 human miRNAs were significantly differentially expressed between colonic tumor tissues and para-cancerous tissues. Using microarray analysis in 3 paired CRCs, Lin et al (23) revealed that 28 miRNAs were differentially expressed in CRC with liver metastasis compared with their non-metastatic counterparts.

This study is the first to reveal the miRNA expression profiling of brain metastases from CRC. We selected 3 primary CRCs and 3 brain-metastatic carcinomas for the expression analysis of miRNAs. Our results indicated that an overexpression of miR-145, miR-1, miR-146a, miR-576-5p, miR-126*, HS_287, miR-28-5p, miR-143, miR-199b-5p, miR-199a-5p, miR-10b, miR-22, miR-133b, miR-145*, miR-199a, miR-133a, miR-125b, and a downregulation of miR-31 and HS_170 occurred in brain-metastatic carcinomas. However, previous studies have shown that the expression of miR-143 and miR-145 was downregulated in CRC compared to normal mucosa $(24,25)$. Additionally, miR-31 expression was significantly upregulated in colon tumors relative to hte normal colon tissue $(26,27)$. We propose that a given miRNA may have multiple functions in the processes of carcinogenesis and metastasis.

The quantitative RT-PCR experiments with miR-125b confirmed the expression patterns we found in our microarray experiments. However, Glud et al $(28,29)$ proposed that downregulation of miR-125b in malignant melanoma may increase the metastatic ability of this tumor. Liang et al (30) found that miR-125b inhibited hepatocellular carcinoma cell migration and invasion through the suppression of oncogene LIN28B expression. We propose that $\mathrm{miR}-125 \mathrm{~b}$ may have various functions and target genes in different types of cells. Other miRNAs also regulate miR-125b or its target gene.

Our data are consistent with those of previous studies, which revealed that certain miRNAs are involved in tumor invasion and metastasis, such as miR-199a, miR-10b and miR-31. Song et al showed that miR-199a was highly expressed in gastric cancer compared to normal gastric tissues and in metastatic, compared to non-metastatic gastric cancer tissues (31). MiR-199a positively regulated gastric cancer cell proliferation, migration and invasion. Several investigators $(32,33)$ found that miR-10b was highly expressed in metastatic breast cancer cells and positively regulated cell migration and invasion. miR-10b also positively regulated the metastasis of nasopharyngeal carcinoma (34). The expression of miR-31 correlated inversely with metastasis in human breast cancer patients. Loss of miR-31 was associated with cancer progression and metastasis (35-37).

By examining the miRNA expression of primary CRC and brain metastases, we were able to identify miRNAs associated with the brain metastasis of human CRC. Validation of miRNA expression signatures in a larger series is required to improve the understanding of the metastatic process of CRC. Several noteworthy candidate miRNAs may be potential diagnostic markers and therapeutic targets.

\section{Acknowledgements}

This study was supported by a grant from the 'Star of Century' Foundation of Fudan University. We thank Shanghai Biotechnology Corporation for the technical support and valuable discussion.

\section{References}

1. Sung JJ, Lau JY, Young GP, et al: Asia Pacific consensus recommendations for colorectal cancer screening. Gut 57: 1166-1176, 2008.

2. Mongan JP, Fadul CE, Cole BF, et al: Brain metastases from colorectal cancer: risk factors, incidence, and the possible role of chemokines. Clin Colorectal Cancer 8: 100-105, 2009.

3. Mahmoud N and Bullard Dunn K: Metastasectomy for stage IV colorectal cancer. Dis Colon Rectum 53: 1080-1092, 2010.

4. Tan WS, Ho KS and Eu KW: Brain metastases in colorectal cancers. World J Surg 33: 817-821, 2009.

5. Koehler A, Bataille F, Schmid C, et al: Gene expression profiling of colorectal cancer and metastases divides tumours according to their clinicopathological stage. J Pathol 204: 65-74, 2004.

6. Koh KH, Rhee H, Kang HJ, et al: Differential gene expression profiles of metastases in paired primary and metastatic colorectal carcinomas. Oncology 75: 92-101, 2008.

7. Bernards R and Weinberg RA: A progression puzzle. Nature 418: $823,2002$.

8. Nicoloso MS, Spizzo R, Shimizu M, Rossi S and Calin GA: MicroRNAs - the micro steering wheel of tumour metastases. Nat Rev Cancer 9: 293-302, 2009.

9. Lagos-Quintana M, Rauhut R, Lendeckel W and Tuschl T: Identification of novel genes coding for small expressed RNAs. Science 294: 853-858, 2001.

10. Cheng AM, Byrom MW, Shelton J and Ford LP: Antisense inhibition of human miRNAs and indications for an involvement of miRNA in cell growth and apoptosis. Nucleic Acids Res 33: 1290-1297, 2005

11. Wang YX, Zhang XY, Zhang BF, Yang CQ, Chen XM and Gao HJ: Initial study of microRNA expression profiles of colonic cancer without lymph node metastasis. J Dig Dis 11: 50-54, 2010.

12. Schetter AJ, Leung SY, Sohn JJ, et al: MicroRNA expression profiles associated with prognosis and therapeutic outcome in colon adenocarcinoma. JAMA 299: 425-436, 2008.

13. Ueda T, Volinia S, Okumura H, et al: Relation between microRNA expression and progression and prognosis of gastric cancer: a microRNA expression analysis. Lancet Oncol 11: 136-146, 2010.

14. Lu J, Getz G, Miska EA, et al: MicroRNA expression profiles classify human cancers. Nature 435: 834-838, 2005.

15. Chaffer CL and Weinberg RA: A perspective on cancer cell metastasis. Science 331: 1559-1564, 2011.

16. Kruser TJ, Chao ST, Elson P, et al: Multidisciplinary management of colorectal brain metastases: a retrospective study. Cancer 113: $158-165,2008$.

17. Aprile G, Zanon E, Tuniz F, et al: Neurosurgical management and postoperative whole-brain radiotherapy for colorectal cancer patients with symptomatic brain metastases. J Cancer Res Clin Oncol 135: 451-457, 2009. 
18. Xiang JB, Gu XD, Sun P, et al: Clinical characteristics and neurosurgical resection of brain metastases from colorectal carcinoma. Chin J Gen Surg (in Chinese) 25: 442-445, 2010

19. Wronski $\mathrm{M}$ and Arbit E: Resection of brain metastases from colorectal carcinoma in 73 patients. Cancer 85: 1677-1685, 1999.

20. Pantaleo MA, Astolfi A, Nannini M, et al: Gene expression profiling of liver metastases from colorectal cancer as potential basis for treatment choice. Br J Cancer 99: 1729-1734, 2008.

21. Zhang H, Li Y and Lai M: The microRNA network and tumor metastasis. Oncogene 29: 937-948, 2010.

22. Huang ZM, Yang J, Shen XY, et al: MicroRNA expression profile in non-cancerous colonic tissue associated with lymph node metastasis of colon cancer. J Dig Dis 10: 188-194, 2009.

23. Lin M, Chen W, Huang J, et al: MicroRNA expression profiles in human colorectal cancers with liver metastases. Oncol Rep 25 739-747, 2011.

24. Arndt GM, Dossey L, Cullen LM, et al: Characterization of global microRNA expression reveals oncogenic potential of miR-145 in metastatic colorectal cancer. BMC Cancer 9: 374, 2009.

25. Ng EK, Tsang WP, Ng SS, et al: MicroRNA-143 targets DNA methyltransferases $3 \mathrm{~A}$ in colorectal cancer. Br J Cancer 101: 699-706, 2009.

26. Wang CJ, Zhou ZG, Wang L, et al: Clinicopathological significance of microRNA-31, -143 and -145 expression in colorectal cancer. Dis Markers 26: 27-34, 2009.

27. Sarver AL, French AJ, Borralho PM, et al: Human colon cancer profiles show differential microRNA expression depending on mismatch repair status and are characteristic of undifferentiated proliferative states. BMC Cancer 9: 401, 2009.

28. Glud M, Manfe V, Biskup E, et al: MicroRNA miR-125b induces senescence in human melanoma cells. Melanoma Res 21 : 253-256, 2011.
29. Glud M, Rossing M,Hother C, et al: Downregulation of miR-125b in metastatic cutaneous malignant melanoma. Melanoma Res 20: 479-484, 2010

30. Liang L, Wong CM, Ying Q, et al: MicroRNA-125b suppressed human liver cancer cell proliferation and metastasis by directly targeting oncogene LIN28B2. Hepatology 52: 1731-1740, 2010.

31. Song G, Zeng H, Li J, et al: miR-199a regulates the tumor suppressor mitogen-activated protein kinase kinase kinase 11 in gastric cancer. Biol Pharm Bull 33: 1822-1827, 2010.

32. Ma L, Teruya-Feldstein J and Weinberg RA: Tumour invasion and metastasis initiated by microRNA-10b in breast cancer. Nature 449: 682-688, 2007.

33. Gee HE, Camps C, Buffa FM, et al: MicroRNA-10b and breast cancer metastasis. Nature 455: E8-E9, 2008.

34. Li G, Wu Z, Peng Y, et al: MicroRNA-10b induced by Epstein-Barr virus-encoded latent membrane protein-1 promotes the metastasis of human nasopharyngeal carcinoma cells. Cancer Lett 299: 29-36, 2010.

35. Valastyan S, Chang A, Benaich N, Reinhardt F and Weinberg A: Concurrent suppression of integrin alpha5, radixin, and RhoA phenocopies the effects of miR-31 on metastasis. Cancer Res 70: 5147-5154, 2010.

36. Valastyan S, Reinhardt F, Benaich N, et al: A pleiotropically acting microRNA, miR-31, inhibits breast cancer metastasis. Cell 137: 1032-1046, 2009.

37. Sossey-Alaoui K, Downs-Kelly E, Das M, Izem L, Tubbs R and Plow EF: WAVE3, an actin remodeling protein, is regulated by the metastasis suppressor microRNA, miR-31, during the invasion-metastasis cascade. Int J Cancer 129: 1331-1343, 2011. 